\title{
Estudo manométrico do esôfago distal de gatos anestesiados com tiopental sódico ${ }^{1}$
}

Manometric study of the distal esophagus of cats anesthetized with sodic thiopental

Carlos Eduardo Meirelles dos Santos ${ }^{2}$, Maria Aparecida Coelho de Arruda Henry ${ }^{3}$,
Sheila Canevesse Rahal ${ }^{4}$, Mauro Masson Lerco

1. Trabalho realizado no Laboratório de Técnica Cirúrgica e Cirurgia Experimental da Faculdade de Medicina de Botucatu - UNESP.

2. Médico Veterinário do Centro Veterinário de Bauru.

3. Professora Titular do Departamento de Cirurgia e Ortopedia da UNESP.

4. Professora Adjunta do Departamento de Cirurgia da Faculdade de Medicina Veterinária e Zootecnia de Botucatu.

5. Professor Assistente do Departamento de Cirurgia e Ortopedia da UNESP.

\section{RESUMO}

OBJETIVO: Obter o padrão de normalidade da pressão e comprimento do esfíncter inferior do esôfago (EIE) em gatos anestesiados com tiopental e analisar a viabilidade prática do anestésico para uso neste tipo de investigação sobre atividade motora do esôfago de felinos.

MÉTODOS : Em 12 gatos anestesiados com tiopental sódico foram realizados estudos manométricos do EIE, com leitura por perfusão em três canais radiais. Foram avaliadas as pressões e comprimentos do EIE.

RESULTADOS: Os valores médios da pressão e comprimento do EIE foram 33,52 ? $12,42 \mathrm{mmHg}$ e 1,6 ? $0,4 \mathrm{~cm}$, respectivamente.

CONCLUSÃO: Foi possível estabelecer valor de referência para a pressão e comprimento do EIE de felinos, com uma contenção e retorno confortáveis para o animal, utilizando o tiopental sódico como agente anestésico.

Descritores: Manometria. Esôfago. Gato. Tiopental.

\begin{abstract}
PURPOSE: To obtain the normality standard of the lower esophageal sphincter (LES) of cats anesthetized with tiopental and at analysing the practical viability of the anesthetic use in felines esophagus motor activity investigation.
\end{abstract}


METHODS: Manometric studies of LES were performed in 12 cats anestetized with tiopental with perfusion reading in three radial channels. LES pressures and lengths were measured.

RESULTS: The mean values of LES pressure and lengths were 33,52 ? 12,42 $\mathrm{mmHg}$ and $1,6 ? 0,4 \mathrm{~cm}$ respectively.

CONCLUSION: A LES reference value for felines pressure and length was determined. Acommodation and return were comfortable for the animals with the use of sodic tiopental as an anesthetic agent.

Key words: Manometry. Esophagus. Cat. Tiopental.

\section{Introdução}

O estudo da atividade motora do esôfago é importante para esclarecer a fisiopatologia de afecções esofágicas tais como o megaesôfago ${ }^{1,2,3}$, doença do refluxo gastroesofágico $^{4,5,6}$ e distúrbios da deglutição, que são de importância significativa tanto na medicina humana como na veterinária.

$\mathrm{O}$ arranjo das fibras musculares do esfíncter inferior do esôfago (E I E) do gato e gambá $^{7,8,9}$, é semelhante ao do homem. Entretanto, Rogers et al ${ }^{1}$., observaram que o EIE de cães com acalasia não se comporta como em humanos com esta doença. Estes fatos, aliados à semelhança do $\mathrm{pH}$ gástrico humano e felino ${ }^{9}$ colocam o gato como melhor modelo de estudo ${ }^{10}$, considerando inclusive que é de mais fácil aquisição e manipulação que o gambá.

Diversos autores têm estudado a fisiologia do esôfago felino ${ }^{10,11}$ visando obter o padrão de normalidade para servir de bases em estudos experimentais.

Correnti et al, ${ }^{10}$ estudaram o E I E de 15 gatos anestesiados com quetamina por via intramuscular. Segundo estes autores a pressão média do E I E foi de 28,1 ? 1,4 mmHg. Como as características dos esfíncters e da motilidade do corpo foram semelhantes a vista em humanos, os autores concluíram ser o gato um modelo apropriado para estudo da motilidade esofageana.

Ao determinarem o papel da inervação vagal extrínseca no controle da função do E I $\mathrm{E}$ em quatro gatos, Reynolds et al $^{12}$., encontraram valores normais do esfíncter inferior do esôfago em média, de 58 ? $17 \mathrm{mmHg}$. Os animais foram anestesiados com quetamina na 
dose de $15 \mathrm{mg} / \mathrm{kg}$ via intramuscular na indução e $10 \mathrm{mg} / \mathrm{kg}$ via intramuscular a cada 30 a 60 minutos de manutenção.

Preiksatis et $\mathrm{al}^{7}$ concluíram, ao avaliarem quatro gatos, que o E I E dessa espécie, semelhante ao dos humanos, apresenta assimetria radial. Os autores observaram também redução significativa da pressão no E I E após injeção de atropina.

Ao efetuarem experimento sobre reversibilidade da motilidade do esôfago em 22 gatos, Schneider et al ${ }^{2}$, observaram valores da pressão no E I E variando entre 31 e 47 mmHg, com média de $37 \mathrm{mmHg}$, como padrão de normalidade na zona de alta pressão deste animal. A manometria esofágica foi efetuada com os animais anestesiados pela quetamina, aplicada via intramuscular na dose de $30 \mathrm{mg}$.

Tendo como argumento a preservação do reflexo de deglutição, Zhang et $a^{8}$, utilizaram a anestesia com quetamina na dose de $15 \mathrm{mg} / \mathrm{kg}$ em cinco gatos, avaliando a peristalse esofágica e a função do E I E (47+/- 4 mmHg no grupo controle).

Hashim e Waterman ${ }^{13,14}$ analisaram em gatos, influência do sulfato de atropina, acepromazina, tiopental, propofol, quetamina, e a associação sulfato de atropina xylazina e quetamina, sobre a pressão e comprimento do E I E. Os autores observaram que todos os fármacos diminuem a pressão do $\mathrm{E} \mathrm{I} \mathrm{E}$, sendo que a quetamina apresentou efeito menos intenso, pois os gatos preservaram reflexo de deglutição e pedalagem.

Visto que no exame manométrico é imprescindível a correta e confortável imobilização do animal na posição supina ${ }^{4,10}$ ou dorsal, para a passagem da sonda por via nasal ${ }^{10}$, ou oral, ${ }^{4}$ o presente trabalho foi realizado com o objetivo de se obter o padrão de normalidade da pressão e comprimento do E I E em gatos anestesiados com tiopental, bem como analisar a viabilidade prática do anestésico para o uso neste tipo de experimentação manométrica e o conforto e segurança para animais anestesiados.

\section{Métodos}

Foram estudados 12 gatos SRD, sendo sete fêmeas e cinco machos, com idades variando entre 2 e 12 meses e pesos entre 1,0 e 3,5 kg. Após jejum de 12 horas os animais foram anestesiados com tiopental sódico, na dose inicial de $30 \mathrm{mg} / \mathrm{kg}$ por via venosa, em pequenos bolus vagarosa e continuamente até a obtenção do segundo plano do terceiro 
estágio anestésico, monitorado pela observação da perda da consciência, perda do reflexo interdigital, diminuição do reflexo palpebral e monitoração da freqüência respiratória.

Em seguida estes animais foram submetidos a manometria esofágica e para tal foi utilizada sonda de três vias de polietileno, com 1,5mm de diâmetro externo. A sonda possui três orifícios radiais, permitindo a obtenção de três medidas de pressão no E I E em cada exame manométrico.

As três vias da sonda foram ligadas a transdutores de pressão, sistema de perfusão de água destilada e fisiógrafo de seis canais computadorizado (Figura 1).

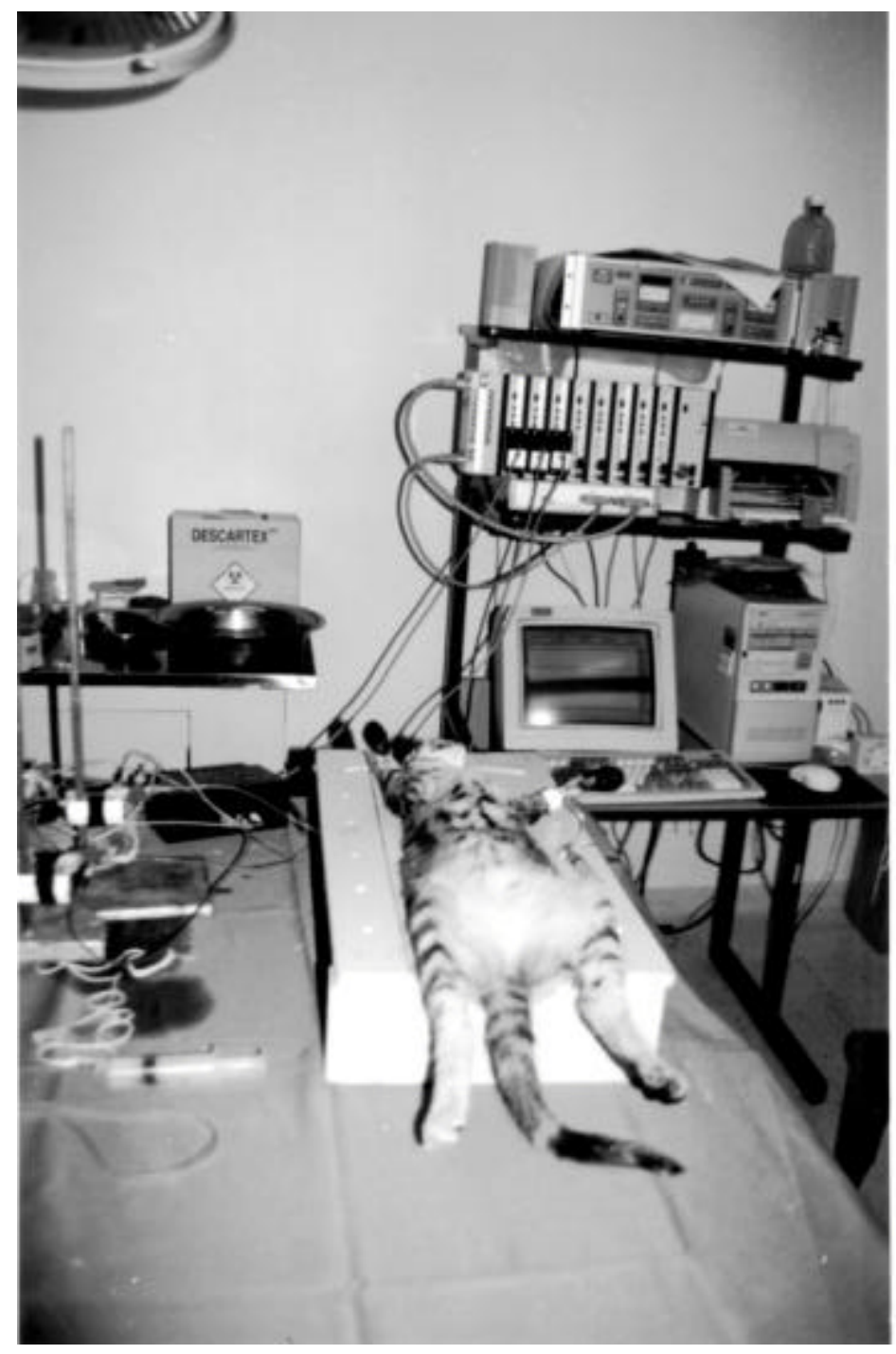

FIGURA 1 - Gato anestesiado durante manometria esofágica computadorizada. 
A eletromanometria foi realizada segundo técnica de puxada intermitente da sonda, segundo padronização do Laboratório de Técnica Cirúrgica e Cirurgia Experimental da Faculdade de Medicina de Botucatu-UNESP; ${ }^{15,16,17}$. Após a introdução no estômago, a sonda foi tracionada de meio em meio centímetro até atingir o E I E e corpo esofágico. A eletromanometria permitiu o estudo da amplitude da pressão do E I E (mmHg) e comprimento do E I E (cm) nos canais 1, 2 e 3 (Figura 2).

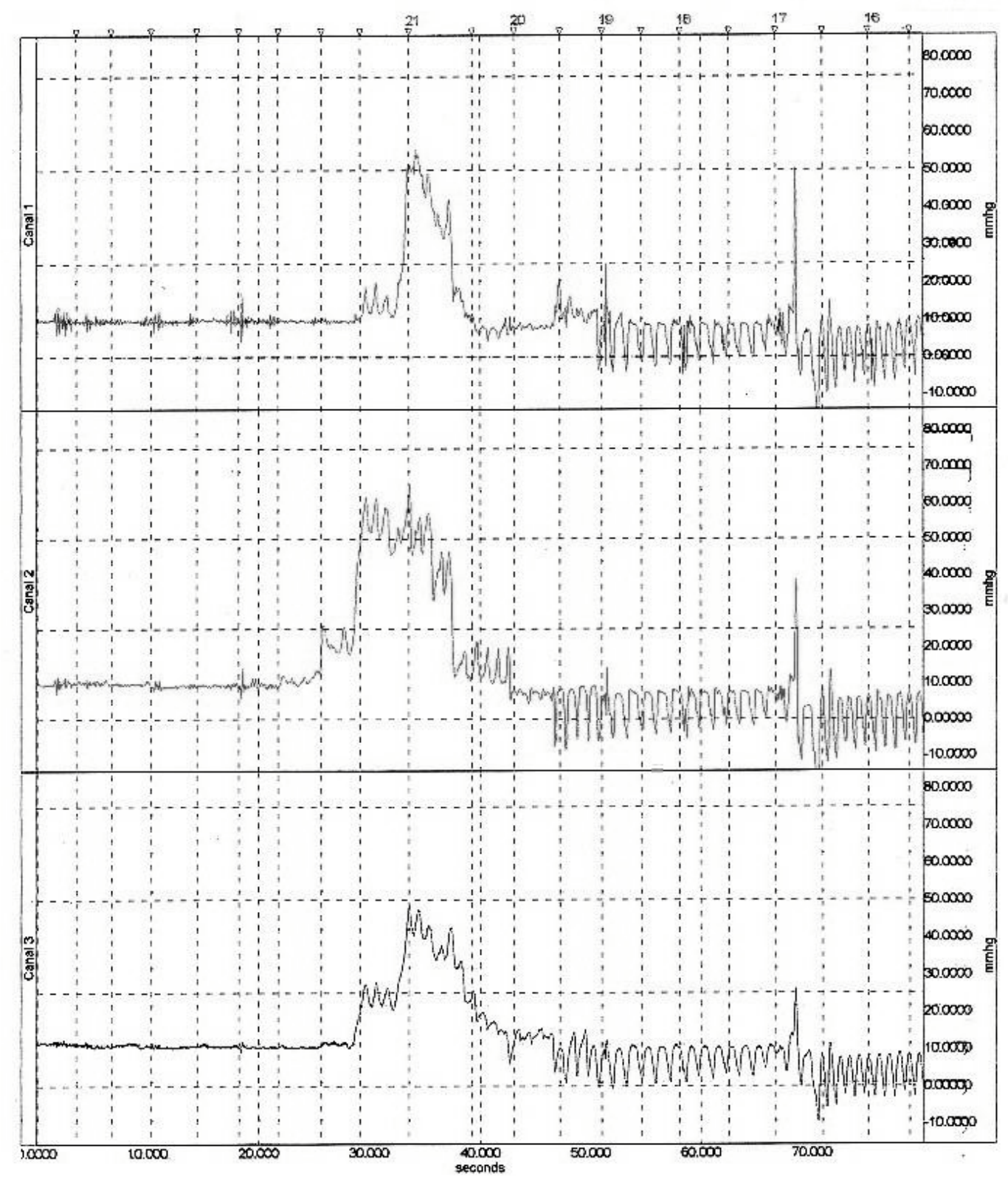

FIGURA 2 - Traçado manométrico mostrando o esfíncter inferior do esôfago nos 3 canais de registro. 
Após o término do exame, os animais foram observados até o retorno anestésico, que ocorria após duas a três horas do início do experimento.

Sendo o objetivo do trabalho a obtenção do padrão de normalidade, o procedimento estatístico utilizado limitou-se ao estudo das médias, desvios padrão e coeficiente de variabilidade das duas variáveis (pressão e comprimento do EIE).

\section{Resultados}

A técnica anestésica utilizada permitiu eficaz contenção dos animais, não tendo sido observado sinais de reflexos contrários a passagem do cateter. A abolição da deglutição foi dose dependente.

Na Tabela 1 estão contidos os valores da pressão no E I E (mmHg) observados nos 3 canais de registro, a média de cada animal, desvio padrão e coeficiente de variabilidade.

TABELA 1 - Amplitude da pressão no E I E de gatos anestesiados com tiopental (mmHg).

\section{CANAL DE REGISTRO}

$\begin{array}{ccccc}\text { Gato } \mathrm{N}^{\circ} & \mathrm{A} & \mathrm{B} & \mathrm{C} & \text { MÉDIA } \\ 1 & 42 & 22 & 34 & 32,66 \\ 2 & 12 & 30 & 30 & 24,00 \\ 3 & 25 & 60 & 55 & 46,66 \\ 4 & 8 & 32 & 33 & 24,33 \\ 5 & 14 & 25 & 11 & 16,66 \\ 6 & 13 & 32 & 19 & 21,33 \\ 7 & 17 & 28 & 24 & 23,00 \\ 8 & 35 & 33 & 61 & 43,00 \\ 9 & 30 & 50 & 38 & 39,33 \\ 10 & 23 & 26 & 55 & 34,66 \\ 11 & 60 & 60 & 58 & 59,33 \\ 12 & 46 & 40 & 26 & 37,33 \\ \text { Média } & & & & 33,52\end{array}$


Menor valor 16,66

Maior valor 59,33 Desvio padrão 12,42

Coeficiente de variabilidade $37 \%$

Os valores do comprimento de E I E $(\mathrm{cm})$ nos três canais de registro, a média de cada animal, desvio padrão e coeficiente de variabilidade estão na Tabela 2.

TABELA 2 - Comprimento do E I E (cm) de gatos anestesiados com tiopental.

CANAL DE REGISTRO

Gato $\mathrm{n}^{\mathrm{o}}$

$\begin{array}{ccccc}1 & 2,0 & 2,0 & 2,0 & 2,0 \\ 2 & 1,0 & 1,0 & 1,5 & 1,2 \\ 3 & 1,5 & 1,5 & 1,0 & 1,3 \\ 4 & 2,0 & 2,0 & 2,0 & 2,0 \\ 5 & 2,0 & 2,0 & 2,0 & 2,0 \\ 6 & 2,0 & 2,0 & 2,5 & 2,2 \\ 7 & 1,5 & 1,0 & 2,0 & 1,5 \\ 8 & 2,0 & 1,5 & 1,5 & 1,5 \\ 9 & 1,5 & 1,5 & 1,5 & 1,5 \\ 10 & 1,5 & 1,0 & 1,0 & 1,0 \\ 11 & 1,0 & 1,5 & 1,0 & 1,2 \\ 12 & 1,0 & 1,0 & 1,5 & 1,2 \\ \text { Média } & & & & 1,6 \\ \text { mor valor } & & & & 1,0 \\ \text { aior valor } & & & & 2,5\end{array}$


Desvio padrão

0,4

Coeficiente de variabilidade

$25 \%$

\section{Discussão}

O presente trabalho foi realizado com o objetivo de estudar o EIE de gatos anestesiados com tiopental sódico, visando a obtenção de um padrão de normalidade, que será utilizado em futuras pesquisas no Laboratório de Técnica Cirúrgica e Cirurgia Experimental da Faculdade de Medicina de Botucatu (UNESP) e analisar a viabilidade do tionembutal sódico como anestésicos nestes experimentos.

A atividade motora do esôfago tem sido objeto de estudo em nosso laboratório, utilizando o cão ${ }^{15,16}$, o coelho ${ }^{17,18}$ e o gambá ${ }^{19}$ como animais de experimentação. Entretanto das espécies estudadas apenas o gambá apresenta a distribuição das técnicas musculares semelhantes à do homem ${ }^{20}$. Todavia, o gambá apresenta algumas desvantagens, pois sua obtenção é difícil e os biotérios não dispões de rotina estes marsupiais. Além disso este animal é bastante agressivo e constitui reservatório natural de alguns protozoários (Tripanossoma cruzi), fatos que contribuem para o temor da equipe encarregada de manipula-lo.

Trabalhos recentes têm demonstrado que o gato constitui bom modelo experimental para estudo da motilidade esofageana ${ }^{2,4,5,8,10}$. O estudo da amplitude da pressão do E I E em gatos realizado por vários autores (Tabela 3), demonstra valores médios deste atributo variando entre $22,1 ? 3,6 \mathrm{mmHg}$ e $58 ? 17 \mathrm{mmHg}$. Os autores acima referidos utilizaram a quetamina como agente anestésico, administrada por via intra muscular. 
TABELA 3 - Amplitude da pressão no E I e (mmHg) de gatos anestesiados com quetamina.

\section{Autor}

Eastwood et $\mathrm{al}^{21}$

Reynolds et $\mathrm{al}^{12}$

Correnti et $\mathrm{al}^{10}$

Preiksaitis et $\mathrm{al}^{7}$

Schneider et $\mathrm{al}^{2}$

Zhang et al ${ }^{8}$
Pressão no E I E

$32 ? 5,6$

$58 ? 17$

$28,1 ? 1,4$

$22,1 ? 3,6$

37

$47 ? 4$

Nesta pesquisa utilizou-se o tiopental para anestesiar os animais e foi obtido como valor médio da pressão do E I E 33,52 ? 12,42 mmHg. Os resultados observados neste trabalho foram semelhantes aos observados por Eastwood et $\mathrm{al}^{21}$, Correnti et $\mathrm{al}^{10}$, Preiksaites et $\mathrm{al}^{7}$ e Schneider et $\mathrm{al}^{2}$. Apenas dois autores ${ }^{8,12}$ observaram valores mais elevados que os obtidos na presente investigação.

Com relação ao comprimento do EIE, observou-se nesta pesquisa o valor médio de $1,6 ? \quad 0,4 \mathrm{~cm}$. Não foi encontrado na literatura trabalho realizado em felino e estudando este parâmetro, fato que inviabiliza a comparação de nossos resultados com os de outros autores.

Assim, acredita-se que o uso do tiopental como agente anestésico dos gatos apresenta vantagens em relação a quetamina, pois os animais da presente investigação permaneceram relaxados durante todo o experimento, em plano anestésico mais uniforme e contínuo, o retorno anestésico foi suave e rápido, além de índice de óbito nulo.

O encontro de valores de pressão do E I E semelhante ao observado na literatura e ausência de efeitos colaterais com o uso do tiopental nos levam a conclusão de que esta droga pode ser utilizada com segurança nos estudos da motilidade esofágica, usando o gato como modelo experimental.

\section{Conclusão}


O valor de referência para a pressão e comprimento do EIE de felinos foram estabelecidos, com uma contenção e retorno confortáveis para o animal, utilizando o tiopental sódico como agente anestésico.

\section{Referências}

1. Rogers AW, Fenner WR, Robert GS. Eletromyographic and esophagomanometric findings in clinically normal dogs and in dogs with idiophatic megaesophagus. JAVMA. 1979; 174:181-3.

2. Schineider JH, Peters JH, Kirkman E, Bremner CG, Demeester TR. Are the motility abnormalities of achalasia reversible? An experimental outflow obstruction in the feline model. Surgery 1999; 125:498-503.

3. Crema E, Ferreira DA, Silva AA, Ferreira Santos R. Análise comparativa de três tipos de válvulas anti refluxo associada a cirurgia de Heller: estudo experimental em cães. Rev Col Bras Cir 2002;29:249-56.

4. Vicente YAMVA. Estudo manométrico do esôfago distal do gato. Tese de doutorado. Faculdade de Medicina de Ribeirão Preto, 1990.

5. Sifrim D, Silny J, Holloway RH, Janssens JJ. Patters of gas and liquid reflux during transient lower oesophageal sphincter relaxation: a study using intraluminal electrical impendance. Gut 1999; 44:47-54.

6. Henry MACA, Motta DC, Silva RA. Avaliação manométrica do esôfago distal de coelhos submetidos a fundoplicatura total laparotômica e laparoscópica. Arq Gastroenterol 2002; 39:106-10.

7. Preiksaitis HG, Tremblay L, Diamant NE. Cholinergic responses in the cat lower esophageal sphincter show regional variation. Gastroenterology 1994;106-381-8.

8. Zhang X, Tack J, Janssens J, Sifrim DA. Effect of sildenafil, a phosphodiestrerase5 inhibitor, on esophageal peristalsis and lower oesophageal sphincter function in cats. Neurogastroenterol Motil 2001; 13:325-39. 
9. Poorkhalkali HG, Rich I, Jacobson J, Amaral S, Mgliori, C, Chrostec P, Biancani JL, Cabero HF Helander. Chronic oesophagitis in the cat. Scand J Gastroenterol 2001; 9: 904-9.

10. Correnti FS, Little AG, Calleja IJ, Skinner DB. Manometric evaluation of the feline esophagus. J Surg Res 1986; 41:312-8.

11. Dodds WJ, Stezwart ET, Hodges D, Zboralske E F. Movement of feline esophagus associated with respiration and peristalses. J Clin Invest 1973; 52: 1-13.

12. Reynolds RPE, EL-Shakrawy T, Diamant NE. Lower esophageal sphincter function in the cat: role of central innervation assessed by transient vagal blockade. Am $\mathbf{J}$ Phisiol 1984; G 666-G74.

13. Hashim MA, Waterman AE. Effects of thiopentone, propofol, alphaxalonealphadolone, ketamine and xylazine-ketamine on lower oesophageal sphincter pressure and barrier pressure in cats. Vet Record 1991; 129-137-9.

14. Hashim MA, Waterman AE. Effects of acepromazine, pethidine and atropine premedication on lower oesophageal sphincter pressure and barrier pressure in anaesthetized cats. Vet Record 1993; 133: 158-60.

15. Henry MACA, Leite CVS, Mendes E F. Influência da vagotomia gástrica proximal sobre a zona de alta pressão esôfago-gástrica no cão. Arq Gastroenterol 1988; 25: 188-92.

16. Bretan O, Henry MACA, Cury PR. Techniques in eletromanometry of upper esophageal sphincter. Arch Otolaryngol Head Neck Surg 1990; 116:914-6.

17. Tagliarini JV, Henry MACA, Bretan O. Eletromanometria do esfíncter superior do esôfago antes e após perfusão esofágica com ácido clorídrico.

18. Uvo SAB, Henry MACA. Efeito da laringectomia horizontal associada ou não a esvaziamento cervical e glossectomia total sobre o esfíncter superior do esôfago. Estudo experimental no coelho. Acta Cir Bras 1993; 8:11-4.

19. Henry MACA, Atanasio EYU. Efeito da metoclopamida da ranitidina e do droperidol sobre o esfíncter do esôfago: estudo experimental no gambá (Didelphis albi-ventris). Arq Gastroenterol 1994; 31:103-7.

20. Cohen S, Dimarino SAJ. Mechanism of actian of metoclopramide on opossum lower esophageal sphincter muscle. Gastroenterology 1976; 71:996-8. 
21. Eastwood GL, Donal OC, Richard HH. Experimental esophagitis in cats impairs lower esophageal sphincter pressure. Gastroenterology 1975; 69: 146-53.

Correspondência:

Profa. Maria Aparecida Coelho de Arruda Henry

R. Miguel Ciofi, 200

18607-790 Botucatu - SP

Tel: (14) 3815-2537

Data do recebimento: $22 / 02 / 2004$

Data da revisão: 17/03/2004

Data da aprovação: 04/04/2004

Conflito de interesse: nenhum

Fonte de financiamento: nenhuma

\section{Como citar este artigo:}

Santos CEM, Henry MACA, Rahal SC, Lerco M M. Estudo manométrico do esôfago distal de gatos anestesiados com tiopental sódico. Acta Cir Bras [serial online] 2004 MaioJun;19(3). Disponível em URL: http://www.scielo.br/acb. [também em CD-ROM]. 